
\title{
西
}

\section{Health impact of marine carotenoids}

\author{
Kazuo Miyashita* and Masashi Hosokawa
}

Faculty of Fisheries Sciences, Hokkaido University, 3-1-1 Minato, Hakodate, Hokkaido 041-8611, Japan

${ }^{*}$ Corresponding author: Faculty of Fisheries Sciences, Hokkaido University, 3-1-1 Minato, Hakodate, Hokkaido 041-8611, Japan. Tel., E-mail: kmiya@fish.hokudai.ac.jp

DOI: $10.31665 /$ JFB.2018.1125

Received: January 11, 2018; Revised received \& accepted: January 16, 2018

Citation: Miyashita, K., and Hosokawa, M. (2018). Health impact of marine carotenoids. J. Food Bioact. 1: 31-40.

\begin{abstract}
Marine organisms produce a variety of carotenoids with unique functional groups such as allene, acetylene, acetyl, and hydroxymethyl. Astaxanthin and fucoxanthin are representative marine carotenoids on which numerous studies have been performed. Due to the characteristic conjugated polyene chain and terminal ring structures, both carotenoids can act as strong antioxidants. Major nutritional effects of astaxanthin, such as cardio-, skin-, and ocular-protective effects, are based on its in vivo antioxidant activity. However, the antioxidant activity of fucoxanthin is not largely involved in its characteristic nutritional activity and anti-obesity and anti-diabetic effects. The major molecular mechanisms of both effects involve modulating the expression of related genes and proteins by fucoxanthin metabolites.
\end{abstract}

Keywords: Marine carotenoids; Antioxidant activity; Astaxanthin; Fucoxanthin; Anti-obesity; Anti-diabetes; Rare marine carotenoids.

\section{Introduction}

Carotenoids belong to the tetraterpene family produced by plants, algae, and photosynthetic bacteria. They are the most important pigments responsible for the red and yellow colors of plants and animals. Animals including humans are incapable of synthesizing carotenoids and many are colored by carotenoids derived from their diets. Carotenoids absorbed into animal bodies have a broad range of biological functions, especially in relation to human health. The best known biological function of carotenoids is their established role as provitamin A. Almost 50 carotenoids with $\beta$-ionone end groups, such as $\beta$-carotene, $\alpha$-carotene, and $\beta$-cryptoxanthin, have provitamin A activity. After ingestion, these carotenoids are enzymatically cleaved to form retinol and then retinoic acid (Namitha, and Negi, 2010). Retinoic acid, in its all-trans or 9-cis configuration, is an activator of the retinoic acid receptor (RAR) and the retinoid-X receptor (RXR). By activating these nuclear receptors, retinoic acid can influence the transcription of various retinoidresponse genes, which are related to many types of cellular functions including cell growth, reproduction, and development (De Luca 1991). In addition to this provitamin A activity, carotenoids also play a role in the prevention of non-communicable diseases, such as cardiovascular disease, cancer, and age-related macular de- generation (Cooper et al., 1999; Riboli, and Norat, 2007; Willett, 2001).

Carotenoids of photosynthetic organisms are bound to integral membrane proteins of chloroplasts and chromoplasts and are associated with light-harvesting complexes, where they absorb light across a broad range of the spectral region and transfer the energy to chlorophyll, initiating the photochemical events of photosynthesis (Polívka, and Frank, 2010). Simultaneously, they play important roles in absorbing visible or near-ultraviolet (near-UV) light to protect chlorophyll from light energy damage (Huang et al. 2017). The absorption of light energy is based on the presence of the extended $\pi$-electron system in a conjugated polyene chain of carotenoids (Varela et al., 2015).

The conjugated double bonds system can also quench singlet oxygen $\left({ }^{1} \mathrm{O}_{2}\right)$. Carotenoids are regarded as the most efficient natural quenchers of ${ }^{1} \mathrm{O}_{2}$, and this ability increases with the increasing number of conjugated double bonds (Cantrell et al., 2003; Stahl, and Sies, 1997). ${ }^{1} \mathrm{O}_{2}$ is the main reactive oxygen molecule responsible for UV light-induced photooxidative damage of photosynthetic membranes and animal tissues. The strong ability of carotenoids to quench ${ }^{1} \mathrm{O}_{2}$ has been suggested as the main mechanism by which they afford their beneficial effects on skin protection (Azam et al., 2017; Corinaldesi et al., 2017). Carotenoids can also 
scavenge free radicals. The reactions with free radicals are much more complex than those with ${ }^{1} \mathrm{O}_{2}$ (Böhm et al., 2012). Generally, the electron-rich status of carotenoids makes them more suitable to react with free radicals.

Due to their characteristic chemical structure, marine carotenoids are gaining more attention as possible nutraceuticals that could act as strong antioxidants and reduce the risk of many types of non-communicable diseases; however, there have been relatively few studies on marine carotenoids compared with their terrestrial counterparts such as vegetables and fruits (Böhm et al, 2015; Fassett, and Coombes, 2009; Galasso et al., 2017; Gammone et al., 2015; Namitha, and Negi, 2010; Riccioni, 2009; Stahl, and Sies, 2003). In this contribution the nutritional impact of marine carotenoids, mainly focusing on major marine carotenoids, astaxanthin and fucoxanthin is reviewed.

\section{Marine carotenoids}

In marine environments, carotenoids are widely present in algae, bacteria, and animals. Among the approximately 750 carotenoids reported to date (Galasso et al., 2017), more than 250 are of marine origin. Algae are marine photosynthetic organisms and the primary producers of marine carotenoids. They synthesize $\beta$-carotene de novo from isoprenyl diphosphate via phytoene and lycopene and then alter it to produce other derivatives with an interesting structural diversity (Huang et al., 2017; Stonik, and Stonik, 2015; Takeuchi, 2011) (Fig. 1).

Algae are classified into many divisions, such as Cyanophyta, Glaucophyta, Rhodophyta, Cryptophyta, Heterokontophyta, Haptophyta, Dinophyta, Euglenophyta, Chlorarachniophyta, and Chlorophyta. Their sizes vary greatly. Macroalgae can grow up to more than $50 \mathrm{~m}$ in length while the size of some microalga is less than $1 \mu \mathrm{m}$. Macroalgae, such as seaweeds, are classified into three main groups based on their pigmentation: brown, red, and green seaweeds. Brown (Phaeophyceae) and green (Chlorophyceae) seaweeds belong to Heterokontophyta and Chlorophyta, respectively. Rhodophyta can be divided into two groups, unicellular type and macrophytic type. The former is regarded as micro red algae and the latter as red seaweeds. These macro/microalgae produce a wide variety of carotenoids. Approximately 50 carotenoids are found in these algae (Huang et al., 2017).

The most common carotenoids in algae are $\beta$-carotene and zeaxanthin. They are also recognized as major carotenoids in terrestrial plants. Chlorophyta, including green seaweeds (Chlorophyceae), are biologically close to terrestrial plants. Therefore, they contain $\beta$-carotene, violaxanthin, neoxanthin, and lutein as major carotenoids, which are rich in terrestrial plants. Chlorophyta also produce loroxanthin, siphonaxanthin and prasinoxanthin from lutein (Fig. 1). They contain $\mathrm{CH}_{2} \mathrm{OH}$ as a side chain group and are not found in terrestrial plants. Some algae carotenoids have a unique structure, such as allene $(\mathrm{C}=\mathrm{C}=\mathrm{C})$, acetylene $(\mathrm{C} \equiv \mathrm{C})$, acetylation $\left(-\mathrm{O}-\mathrm{CO}-\mathrm{CH}_{3}\right)$, and others (Dembitsky, and Maoka, 2007) (Fig. 1).

Fucoxanthin (Fig. 1) is a representative allene carotenoid found in Rhodophyta, Heterokontophyta, Haptophyta, and Dinophyta. Fucoxanthin is the most abundant carotenoid and contributes more than $10 \%$ of the estimated total carotenoids in nature (Matsuno, 2001). Other allenic carotenoids are 19'-acyloxyfucoxanthin in Haptophyta and Dinophyta, peridinin in Dinophyta, and 9'-cis neoxanthin in green seaweeds (Chlorophyceae). Acetylene carotenoids are found only in algae. Major acetylene carotenoids are alloxanthin, crocoxanthin and monadoxanthin in Cryptophyta, and diadinoxanthin and diatoxanthin in Heterokontophyta, Haptophyta, Dinophyta and Euglenophyta. Acetylated carotenoids, such as fucoxanthin, peridinin and dinoxanthin, are also mainly found in algae.

Carotenoids found in the body of marine animals are either the result of the direct accumulation of dietary carotenoids or are partly modified through metabolic reactions. These carotenoids are responsible for the color of many important marine animals including fish, shellfish, shrimp, and crab. A variety of unique carotenoids have been reported from marine animals, such as Porifera (marine sponges), Coelenterata (sea anemones), Mollusca (mollusks), Arthropoda (crustaceans), Echinodermata (echinoderms), Protochordata (tunicates), and Pisces (fish) (Maoka, 2011). Most carotenoids are metabolites of dietary carotenoids, such as $\beta$-carotene, fucoxanthin, peridinin, diatoxanthin, alloxanthin, and astaxanthin, among others.

Astaxanthin is produced by microalgae such as Haematococcus pluvialis. It is also rich in some marine animals. Many crustaceans can synthesize astaxanthin from $\beta$-carotene that is ingested from dietary algae. In addition, Cyprinidae fish can synthesize astaxanthin from dietary zeaxanthin by oxidative metabolic conversion. Alternatively, Perciformes and Salmonidae fish cannot synthesize astaxanthin from other carotenoids. They accumulate astaxanthin from dietary crustacean zooplankton.

Among the many types of marine carotenoids, much attention has been paid to astaxanthin and fucoxanthin because both carotenoids are abundant in food and show strong antioxidant activity. Oxidative stress and chronic inflammation are the main pathophysiological factors contributing to the development of non-communicable diseases, such as diabetes, hypertension, atherosclerosis, and cardiovascular diseases. In contrast, carotenoids can protect cells, tissues and other structures such as lipoproteins against oxidative damage, and then reduce the risk of many types of diseases. For example, astaxanthin can reduce cardiovascular disease (Fassett, and Coombes, 2009; Fredric et al., 2011) due to its strong antioxidant activity. Artery disorders advance from local redox disequilibrium to vascular endothelial dysfunction, inflammation, excessive vascular remodeling, and finally atherosclerosis (Gori, and Nzel, 2011). Alternatively, astaxanthin has a strong ability to quench ${ }^{1} \mathrm{O}_{2}$ and scavenge free radicals, contributing to the regulation of the redox balance (Ambati et al., 2014; Gammone, and D'Orazio, 2015). The antioxidant activity of astaxanthin can also be responsible for preserving membrane structure, reducing low-density lipoprotein levels, enhancing immune system function and regulating gene expression (Ambati et al., 2014).

In addition to the protective effect of carotenoids against the deregulation of redox balance in biological systems, some marine carotenoids such as fucoxanthin are capable of altering the patterns of gene and protein expression and cell function with a specific and important nutritional and bio-functional impact on the body (Miyashita, and Hosokawa, 2017). These modulation effects of carotenoids are based on other mechanisms of action that are independent of their antioxidant properties.

\subsection{Astaxanthin}

Commonly found carotenoids from fish are tunaxanthin in yellow fish, astaxanthin in redfish, zeaxanthin in anchovies, flatfish and shark, tunaxanthin, lutein and zeaxanthin in brackish water fish and lutein and zeaxanthin in freshwater fish (Goodwin, 1986; Matsuno, 2001). Crustaceans, such as shrimp, prawn, lobster, krill and crab, contain astaxanthin as their main pigment present in free forms, esterified or as a form bound to macromolecules such as protein or 


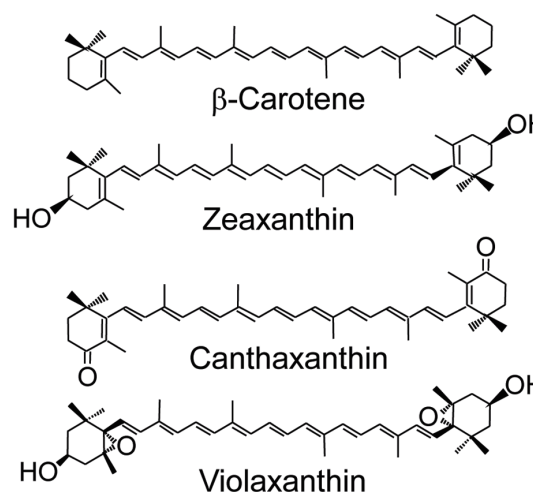

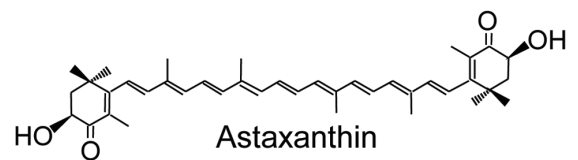

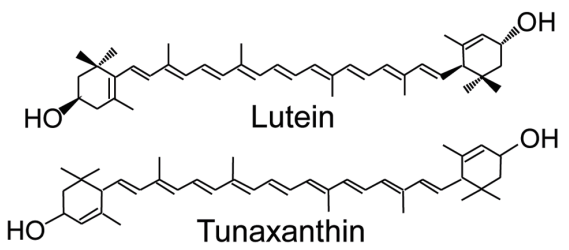

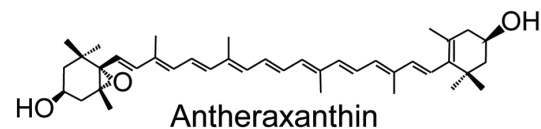

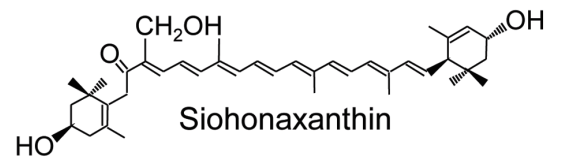

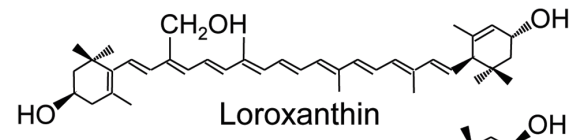

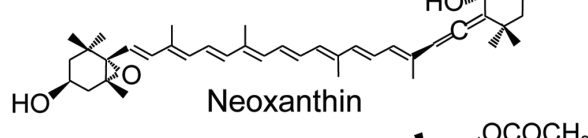

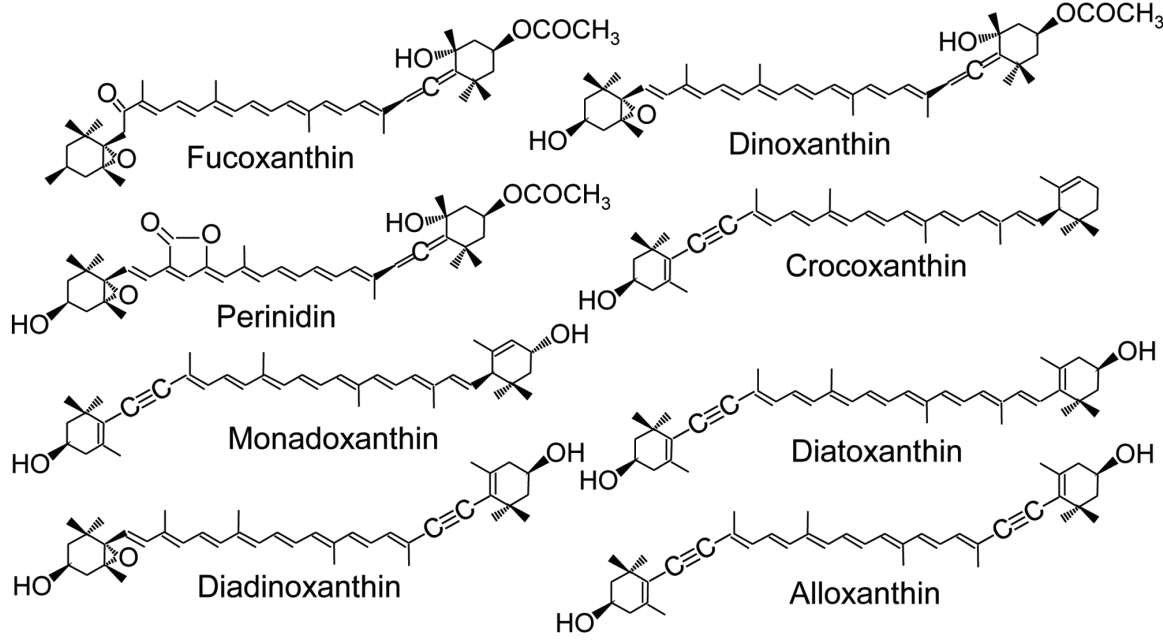

Figure 1. Structure of major marine carotenoids.

chitin. Crustaceans absorb the pigments from the diet and deposit them or transfer them metabolically to keto or hydroxy derivatives (Davies, 1985). Astaxanthin and its esters are the main pigments in crustaceans irrespective of the species and the environment from which they are harvested (Sachindra et al., 2005). Overall, astaxanthin is the main carotenoid found in aquatic animals.

Although the reactions of carotenoids with free radicals are much more complex than with ${ }^{1} \mathrm{O}_{2}$, several studies have suggested the strong relationship between the polarity of an ionone ring and the free radical scavenging ability of carotenoids (Galasso et al., 2017). A study using an 2,2'-azinobis-3-ethylbenzo thizoline6-sulphonate radical cation showed an increase in free radical trapping activity of carotenoids with the presence of functional groups in terminal rings as seen in astaxanthin and fucoxanthin (Miller et al., 1996). Carotenoids bearing terminal carbonyl groups conjugated to a polyene backbone (e.g. astaxanthin and canthaxanthin) were better free radical scavengers (Beutner et al., 2001). The keto function in conjugation with the polyene backbone can stabilize carbon-centered radicals more effectively than the polyene backbone alone (Jackson et al., 2008). Astaxanthin contains a unique molecular structure in the presence of hydroxyl and keto moieties on each ionone ring, which is responsible for the high radical scavenging activity.

Due to the above chemical property, astaxanthin is among the strongest antioxidant compared to other carotenoids (Corinaldesi et al., 2017; Focsan et al., 2017; Gammone et al., 2015; HigueraCiapara et al., 2006). Astaxanthin is more effective than $\beta$-carotene in preventing fatty acid peroxidation in chemical solutions (Terao, 1989) and delaying lipid peroxidation in membrane models (Lim et al., 1992). Astaxanthin has a stronger ability to destroy free radicals than other carotenoids (Lawlor, and O'Brien, 1995). Goto et al. (2001) examined the effects of $\beta$-carotene and astaxanthin on $\mathrm{ADP} / \mathrm{Fe}^{2+}$ induced lipid peroxidation in liposomes under various conditions and found that astaxanthin had a higher antioxidant activity than $\beta$-carotene. They further showed that astaxanthin trapped radicals not only at the conjugated polyene chain but also 


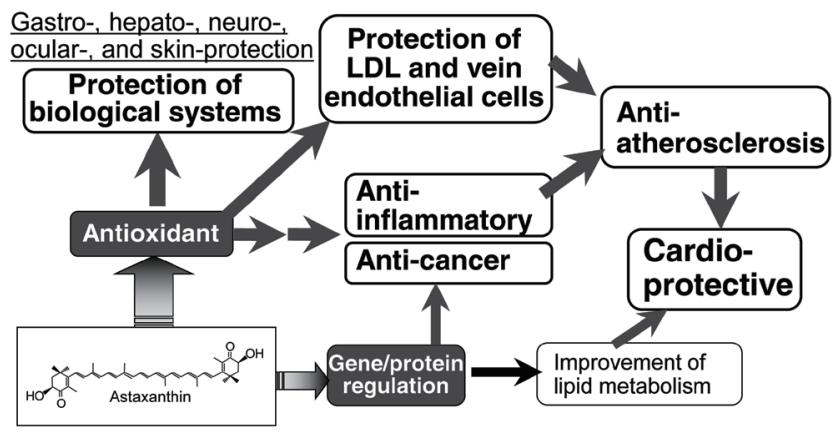

Figure 2. Possible mechanism of nutritional activity of astaxanthin.

in the terminal ring moiety in which the hydrogen atom at the $\mathrm{C} 3$ methine was suggested to be the radical trapping site. This trapping activity of the terminal ring is due to the higher antioxidant activity of astaxanthin at the surface and inside the phospholipid membrane. Comparison of the antioxidant activity of carotenoids in liposomes indicated that astaxanthin- $\beta$-glucoside showed the strongest antioxidant activity, followed by astaxanthin, zeaxanthin, and $\beta$-carotene, although there was no significant difference in their antioxidant activity in chloroform solution (Matsushita et al., 2000). This difference was not only dependent on the ability of carotenoid to scavenge free radicals, but on their location and orientation in the liposome bilayers.

Fluorometric assays showed that the relative reactivities of astaxanthin, lutein, lycopene, $\alpha$-carotene, $\beta$-carotene, and $\alpha$-tocopherol toward peroxyl radicals were $1.3,0.4,0.4,0.5,0.2$, and 0.9 , respectively (Naguib, 2000). Analysis of hydroperoxide formation in membrane lipids showed that apolar carotenoids, such as lycopene and $\beta$-carotene, could disorder the membrane bilayer and showed a potent pro-oxidant effect with an $85 \%$ increase in lipid hydroperoxide levels, whereas astaxanthin preserved membrane structure and exhibited significant antioxidant activity with a $40 \%$ decrease in lipid hydroperoxide levels (McNulty et al., 2007). In the comparative study on the effect of dietary microalgae rich in $\beta$-carotene, lutein, and astaxanthin (Ranga Rao et al., 2010), astaxanthin showed a better bioavailability compared to the other two carotenoids in a rat model. In addition, a higher activity of antioxidant enzymes was found in rats fed an astaxanthin-rich diet.

In vivo antioxidant activity of astaxanthin is an underlying mechanism for its potent beneficial health effects, such as gastro-, hepato-, neuro-, ocular-, and skin-protective effects (Ambati et al., 2014; Chen, and Kotani, 2016; Grimmig et al., 2017; Yuan et al., 2011; Wu et al., 2015) (Fig. 2). Other nutritional impacts of astaxanthin on the cardiovascular system, inflammation, diabetes, and cancer are also strongly related to its antioxidant activity. For example, human skin is damaged by active oxygen species, especially ${ }^{1} \mathrm{O}_{2}$, which can be produced by $\mathrm{UV}$ irradiation. Astaxanthin can effectively quench ${ }^{1} \mathrm{O}_{2}$ to protect the skin.

The efficacy of the deactivation of ${ }^{1} \mathrm{O}_{2}$ by carotenoids increased with the number of conjugated double bonds. Astaxanthin has 13 conjugated double bonds due to the presence of two conjugated keto groups (Fig. 1), which is higher than those of other major carotenoids, such as lutein (10), lycopene and $\beta$-carotene (11). The strong ability of astaxanthin to quench ${ }^{1} \mathrm{O}_{2}$ has been considered as the mechanism of protecting biological tissues, especially skin, against oxidative damage (Ambati et al., 2014; Yuan et al., 2011; Gammone et al., 2015). Significant and permanent skin damage can occur by solar UV, especially UVA, irradiation (Bossi et al., 2008). UVA can penetrate deeper into the epidermis and dermis of the skin. Exposure of the skin to UVA generates reactive oxygen species, mainly ${ }^{1} \mathrm{O}_{2}$, which damages DNA, lipids and proteins, leading to cellular responses such as modified gene expression. Astaxanthin has photoprotective effects on human skin fibroblasts against UVA damage (Camera, et al., 2008; Lyons et al., 2002). The damage caused by the exposure of skin to UVA up-regulates skin fibroblast elastase and matrix-metalloproteinase-1 in dermal fibroblasts. Astaxanthin inhibits the UVA-induced expression of both enzymes (Suganuma et al., 2010).

There have been several studies on the relationship between the antioxidant activity of astaxanthin and the prevention of cardiovascular disease (Fassett, and Coombes, 2009; Fredric et al., 2008; Kishimoto et al., 2016; Yuan et al., 2011; Visioli, and Artaria, 2017) (Fig. 2). Astaxanthin can prevent atherosclerosis, a major cause of cardiovascular diseases. This effect of astaxanthin is due to the protection of low density lipoprotein and vein endothelial cells against oxidative injury and dysfunction, which are believed to be particularly important for the onset and progression of atherosclerosis. Chronic inflammation contributes to atherosclerosis. Astaxanthin can reduce inflammatory markers in several cell lines and animal models (Visioli, and Artaria, 2017). In all cases, the anti-inflammatory activity of astaxanthin has been predominantly ascribed to its antioxidant activity. Astaxanthin has also been reported to improve dyslipidemia in animal models (Hussein et al., 2007; Ikeuchi et al., 2007; Yang et al., 2011). This effect of astaxanthin is not directly related to its antioxidant activity but instead based on the regulation of key molecules responsible for lipid metabolism (Iizuka et al., 2012). Accumulating evidence suggests that astaxanthin can exert preventive actions against cardiovascular disease via its potential to improve oxidative stress, inflammation, and lipid metabolism. Other beneficial health effects of astaxanthin, such as anti-inflammatory, anti-cancer, and immuno-modulation activities, are also closely related to its strong antioxidant activity.

\subsection{Fucoxanthin}

Because its chemical structure contains an unusual allenic bond, a 5,6-monoepoxide, two hydroxyl groups, a long central chain of conjugated double bonds, and a carbonyl group conjugated with the polyene chain (Fig. 1), fucoxanthin shows strong antioxidant activity (Nishino, 1998; Nomura et al., 1997; Takashima et al., 2012; Yan et al., 1999). When 19 natural carotenoids were screened for their structure-function relationship with respect to radical scavenging activity using human promyelocytic HL-60 cells, the presence of an allenic bond, as seen in fucoxanthin, has been revealed as an important factor for inhibiting superoxide and NO generation (Murakami et al., 2000). Sachindra et al. (2007) analyzed the antioxidant activity of fucoxanthin and found that the hydroxyl radical scavenging activity of fucoxanthin was 13.5 times higher than that of $\alpha$-tocopherol. The antioxidant activity of fucoxanthin is related to its nutritional effects, such as skin- (Heo, and Jeon, 2009; Zheng et al., 2013; Zheng et al., 2014), hepato(Airanthi et al., 2011; Kaneko et al., 2013), and ocular- (Liu et al., 2016) protection (Fig. 3).

Fucoxanthin has been reported to ameliorate cardiovascular dysfunction mainly through its antioxidant and anti-inflammatory effect (Grasa-López et al., 2016). Alternatively, fucoxanthin is known to have an anti-obesity effect. Because obesity increases the risk of cardiovascular diseases and induces inflammation, the cardio-protective and anti-inflammatory ability of fucoxanthin is strongly related to its anti-obesity activity. The anti-obesity effect of fucoxanthin has been reported in cellular models, animals, and humans (Gammone, and D'Orazio, 2015; Hu et al., 2016; Maeda, 


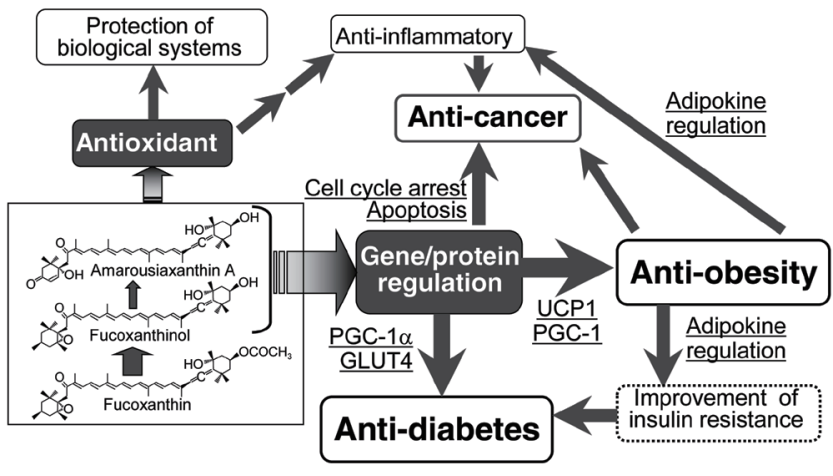

Figure 3. Possible mechanism of nutritional activity of fucoxanthin.

2015; Muradian et al., 2015; Zhang et al., 2015). When various carotenoids were screened for potential suppressive effects on adipocyte differentiation (Maeda et al., 2006; Okada et al., 2008; Yim et al., 2011), only fucoxanthin, neoxanthin, and two fucoxanthin metabolites (fucoxanthinol and amarouciaxanthin A) showed an encouraging suppressive effect on the differentiation of 3T3-L1 adipose cells and intercellular lipid accumulation. This effect was accompanied by a decrease in glycerol-3-phosphate dehydrogenase activity and down-regulation of adipogenic genes, such as peroxisome proliferator-activated receptor $\gamma$ (PPAR $\gamma)$, CCAAT/ enhancer binding protein $\alpha(\mathrm{C} / \mathrm{EBPR} \alpha)$, and sterol regulatory element-binding protein 1c (SREBP1c) (Kang et al., 2011; Maeda et al., 2006; Okada et al., 2008; Yim et al., 2011).

The first report on the anti-obesity effect of fucoxanthin was identified using brown seaweed lipids containing $10 \%$ fucoxanthin (Maeda et al., 2005). After, the anti-obesity effect of fucoxanthin was confirmed using a mouse model of obesity-diabetes (Beppu et al, 2013; Hosokawa et al., 2010; Maeda et al., 2007a; Maeda et al., 2007b) and normal animals fed a high-fat diet (Hu et al., 2012; Kang et al., 2012; Maeda et al., 2009; Woo et al., 2009). A comparative study using an obese/diabetes KK- $A^{\mathrm{y}}$ mouse model and wildtype $\mathrm{C} 57 \mathrm{BL} / 6 \mathrm{~J}$ mice indicated that fucoxanthin attenuates excess fat accumulation in the abdominal white adipose tissue (WAT) of the obese $\mathrm{KK}-A^{\mathrm{y}}$ mice while no effect was found in the $\mathrm{C} 57 \mathrm{BL} / 6 \mathrm{~J}$ mice fed a normal-fat diet (Hosokawa et al., 2010). Alternatively, feeding fucoxanthin to the mice significantly suppressed the $a b-$ dominal WAT weight of C57BL/6J mice fed a high-fat diet to the same level of that found in the normal dietary group (Maeda et al., 2009). Therefore, the suppressive effect of fucoxanthin on WAT weight gain is specific for adiposity in the development of obesity in mice. This specificity is important for the safe application of fucoxanthin in human obese therapy.

Fucoxanthin supplementation was also tested for weight loss in humans. In a 16-week study of the effects of fucoxanthin in obese, non-diabetic, premenopausal female volunteers (Abidov et al., 2010), $2.4 \mathrm{mg}$ of fucoxanthin intake resulted in a significant decrease in body fat, body weight, liver fat content, and serum triglycerides. The clinical trial also demonstrated an increase in the resting energy expenditure with $>2.4 \mathrm{mg}$ fucoxanthin intake. In a recent study (Hitoe, and Shimoda, 2017), 1 or $3 \mathrm{mg}$ fucoxanthin was administered to male and female Japanese adults with a body mass index (BMI) of more than $25 \mathrm{~kg} / \mathrm{m}^{2}$ for 4 weeks. There was a significant reduction of relative body weight, body mass index and visceral fat area in the $3 \mathrm{mg}$ /day fucoxanthin group compared to the placebo group. In addition, the relative values of total fat mass, subcutaneous fat area, waist circumference, and right thigh circumference were also significantly lower in the $1 \mathrm{mg} /$ day fucox- anthin group than the placebo group.

Although several mechanisms have been proposed for the antiobesity effect of fucoxanthin, the major target of fucoxanthin is adipose tissues, and the main mechanism is adaptive thermogenesis via uncoupling protein 1 (UCP1) induction (Gammone and D’Orazio, 2015; Hu et al., 2016; Miyashita et al., 2011; Muradian et al., 2015; Zhang et al., 2015) (Fig. 3). UCP1 is a key factor in the thermogenic process occurring in brown adipose tissue (BAT) (Nedergaard et al., 2001; Rothwell and Stock, 1979; Smith and Horwitz, 1969) and beige adipocytes in WAT (Brestoff and Artis, 2015; Harms and Seale, 2013; Mueller, 2016). Feeding fucoxanthin to mice increased the BAT weight and induced UCP1 mRNA and protein expression in abdominal WAT (Maeda et al., 2005; Maeda et al., 2007a; Maeda et al., 2007b), showing that the antiobesity effect of fucoxanthin is derived from the up-regulation of thermogenesis through UCP1 expression in both BAT and abdominal WAT. The UCP1 induction in abdominal WAT is due to the activation of beige adipocytes in abdominal WAT (Miyashita, and Hosokawa, 2017).

Fucoxanthin intake can normalize the blood glucose level in an obese/diabetes $\mathrm{KK}-A^{\mathrm{y}}$ mouse model (Maeda et al., 2007b) and in wild-type C57BL/6J mice fed a high-fat diet (Maeda et al., 2009; Park et al., 2011; Woo et al., 2010). Alternatively, fucoxanthin showed little effect on lowering the blood glucose level of wildtype C57BL/6J mice fed a normal diet (Hosokawa et al., 2010). One possible mechanism of the anti-diabetic effect of Fx is regulation of the release of adipokines from abdominal WAT (Fig. 3). Excess fat accumulation in abdominal WAT causes a chronic, lowgrade inflammation in this tissue and then increases the production of pro-inflammatory adipokines. The over-secretion of these adipokines induces insulin resistance. In contrast, fucoxanthin supplementation down-regulates the pro-inflammatory adipokine production and secretion (Hosokawa et al., 2010).

Another mechanism of the anti-diabetic effect of fucoxanthin is the regulation of glucose transporter 4 (GLUT4) (Maeda et al., 2009; Nishikawa et al., 2012) (Fig. 3). With insulin and other stimuli, GLUT4 expression is up-regulated and quickly moves to the plasma membranes from an intracellular location and promotes glucose uptake in skeletal muscle (Huang and Czech, 2007). However, in type 2 diabetes mellitus, insulin signaling is impaired and GLUT4 expression and its translocation is attenuated (Henriksen et al., 1999). Fucoxanthin administration to diabetes animal models completely normalized these perturbations.

\subsection{Other marine carotenoids}

Although novel physiological activity is expected from the chemical structure of marine carotenoids, there has been a limited study on the nutritional effect of these carotenoids, besides astaxanthin and fucoxanthin. Due to their rareness, the nutritional effect of these carotenoids has only been analyzed using cellular models and not animal models. The sea squirt Halocynthia roretzi has a metabolic pathway that converts fucoxanthin to acetylene carotenoids: mytiloxanthin, halocynthiaxanthin, all-trans alloxanthin, 9-cis alloxanthin, all-trans diatoxanthin, and 9-cis diatoxanthin (Fig. 1). Konishi et al. (2006) found an inhibitory effect of halocynthiaxanthin on the growth of HL-60 human leukemia cells in a dose- and time-dependent manner. Furthermore, halocynthiaxanthin induced apoptosis in HL-60 cells, MCF-7 human breast cancer cells, and Caco-2 human colon cancer cells via down-regulation of apoptosis-suppressing protein, Bcl-2. They also reported an anti-inflammatory effect of other acetylene carotenoids from $\mathrm{Hal}$ ocynthia roretzi (Konishi et al., 2008). All-trans alloxanthin, all- 
trans diatoxanthin, and their 9-cis isomers significantly suppressed the expression of pro-inflammatory cytokines in RAW264.7 cells treated with lipopolysaccharide (LPS). The suppressive effects were remarkable compared with those of $\beta$-carotene and zeaxanthin.

The inhibitory effect on cancer cells has been observed with other marine carotenoids. Peridinin (Fig. 1), an acetylene carotenoid found in microalgae (dinoflagellates), induced apoptosis in colorectal adenocarcinoma cells (DLD-1) with chromatin fragmentation and an up-regulation of caspase-9 and caspase-8 (Sugawara et al., 2007). Siphonaxanthin (Fig. 1) also exhibited a strong apoptosis-inducing effect on cancer cells (Sugawara et al., 2014). This keto-carotenoid present in edible green algae showed a powerful inhibitory effect on the viability of human leukemia HL-60 cells. A previous study reported the anti-proliferative effects of 15 carotenoids from food sources on human prostate cancer cells (Kotake-Nara et al., 2001). Among the carotenoids examined, fucoxanthin and neoxanthin showed the highest activity. In contrast, the anti-proliferative effect of siphonaxanthin on HL-60 cells was higher than that of fucoxanthin. This strong activity of siphonaxanthin was accompanied by reduced Bcl-2 expression and subsequent activation of caspase-3 and up-regulation of death receptor 5 (DR5) expression (Ganesan et al. 2011).

\section{Antioxidant activity}

The major mechanism of the antioxidant activity of carotenoids is quenching ${ }^{1} \mathrm{O}_{2}$ and scavenging free radicals. The quenching of ${ }^{1} \mathrm{O}_{2}$ by carotenoids has been attributed mainly to a physical mechanism, where the excess energy of ${ }^{1} \mathrm{O}_{2}$ is transferred to the carotenoid $\left({ }^{1} \mathrm{Car}\right)$. The overall reaction process simply converts the excess energy of ${ }^{1} \mathrm{O}_{2}$ into heat via the carotenoid lowest excited triplet state ( $\left.{ }^{3} \mathrm{Car}\right)$ as follows (Böhm et al., 2012):

$$
\begin{gathered}
{ }^{1} \mathrm{O}_{2}+{ }^{1} \mathrm{Car}^{3} \rightarrow \mathrm{O}_{2}+{ }^{3} \mathrm{Car} \quad \text { (1) } \\
{ }^{3} \mathrm{Car} \rightarrow{ }^{1} \mathrm{Car}+\text { heat }
\end{gathered}
$$

The detailed mechanism for the energy transfer from ${ }^{1} \mathrm{O}_{2}$ to carotenoid (1) is quite complex (Schmidt, 2004), involving singlet and triplet encounter complexes leading to the formation of ${ }^{3} \mathrm{Car}$. The carotenoid with added energy is excited to a triplet state $\left({ }^{3} \mathrm{Car}\right)$ and, upon losing the energy as heat, it relaxes to a singlet state $\left({ }^{1} \mathrm{Car}\right)$ without changing the structure (2).

The ${ }^{1} \mathrm{O}_{2}$ quenching rates of carotenoids are characterized by the rate constant $\mathrm{k}_{\mathrm{q}}$, with larger $\mathrm{k}_{\mathrm{q}}$ values indicating a faster quenching reaction. Their physical quenching rate constant has been reported to be close to the diffusion-controlled rate constant (approximately $10^{10} / \mathrm{M}^{-1} \mathrm{~s}^{-1}$ ) and is $30-100$ times higher than that of $\alpha$-tocopherol $\left(3 \times 10^{8} / \mathrm{M}^{-1} \mathrm{~s}^{-1}\right)$ (Terao et al., 2011). By increasing the number of conjugated double bonds present in the carotenoid molecule, the energies of the excited states decrease, which is reflected in the dependence of the ${ }^{1} \mathrm{O}_{2}$ quenching rate constant on carotenoid chain length. Therefore, the ability of carotenoids to quench ${ }^{1} \mathrm{O}_{2}$ increases with the increasing number of conjugated double bonds (Conn et al., 1991; Edge et al., 1997).

The quenching ability is also affected by other factors such as the chain structure and functional groups of carotenoids, solvent viscosity, and the substrate dispersion system (Conn et al., 1991; Hirayama et al., 1994; Mascio et al., 1991). For example, the number of conjugated double bonds of lycopene and $\beta$-carotene is 11 while that of astaxanthin is 13 due to the presence of two conjugated keto groups. Therefore, astaxanthin generally has the highest ${ }^{1} \mathrm{O}_{2}$ quenching ability among them. However, the quenching rate constants $\left(\mathrm{k}_{\mathrm{q}} / \mathrm{M}^{-1} \mathrm{~s}^{-1}\right)$ of these carotenoids in ethanol/chloroform/ water were $3.1 \times 10^{10}$ for lycopene, $2.4 \times 10^{10}$ for astaxanthin, and $1.4 \times 10^{10}$ for $\beta$-carotene (Mascio et al., 1991).

Fukuzawa et al. (1998) observed that ${ }^{1} \mathrm{O}_{2}$ quenching of carotenoids is $40-80$ times higher than that of $\alpha$-tocopherol in ethanol but only six times higher in liposomes. Cantrell et al. (2003) also reported that lycopene and $\beta$-carotene showed the fastest ${ }^{1} \mathrm{O}_{2}$ quenching rate constants $\left(2.3-2.5 \times 10^{10} / \mathrm{M}^{-1} \mathrm{~s}^{-1}\right)$ with lutein as the least efficient $\left(1.1 \times 10^{10} / \mathrm{M}^{-1} \mathrm{~s}^{-1}\right)$, when the ability of 6 types of dietary carotenoids to quench ${ }^{1} \mathrm{O}_{2}$ was compared in a model membrane system (unilamellar liposomes). In addition to the chemical structure, the ${ }^{1} \mathrm{O}_{2}$ quenching rates of carotenoids in biological systems also depends on factors such as the concentration of carotenoids in membranes, membrane localization of active groups, the solubility of the generation site of ${ }^{1} \mathrm{O}_{2}$ in membranes and the mobility of carotenoids in membranes.

Another role of carotenoids as antioxidants is attributed to the scavenging of a wide range of free radicals, including $\mathrm{CCl}_{3} \mathrm{O}_{2}$, $\mathrm{RSO}_{2} \bullet, \mathrm{NO}_{2} \bullet$ and various aryl-peroxy radicals. The free radicals obtain the electron from other molecules or form an adduct with the other molecule. The electron rich status of carotenoids makes them more suitable for reaction with the free radicals thus avoiding the use of cellular components by the free radicals for reactions. Krinsky and Yeum (2003) reviewed the carotenoid-radical interactions and suggested the following three possible interactions:

Adduct formation :

$\mathrm{Car}+\mathrm{R} \bullet \rightarrow \mathrm{R}-\mathrm{Car} \bullet$

Electron transfer:

$\mathrm{Car}+\mathrm{R} \bullet \rightarrow \mathrm{Car}^{+}+\mathrm{R}^{-}$

Allylic hydrogen abstraction :

$\mathrm{Car}+\mathrm{R} \bullet \rightarrow \mathrm{Car} \bullet+\mathrm{RH}$.

\section{Beneficial health effects of carotenoids}

Reactive oxygen species and free radicals damage biological molecules and impair their function. Many studies have demonstrated that oxidative damage plays a central role in the cause and progression of several types of non-communicable diseases, including inflammatory diseases, cardiovascular diseases, and cancer. Therefore, the beneficial health effects of carotenoids are strongly related to their antioxidant activity. Skin damage is also mainly induced by reactive oxygen species produced after UV irradiation. The cellular oxidative damage includes modified gene expression, activation or inactivation of kinase-dependent regulatory pathways, immune and inflammatory events, and the induction of apoptosis. The ability of carotenoids to quench ${ }^{1} \mathrm{O}_{2}$ and scavenge free radicals has been suggested as the main mechanism by which they afford their beneficial health effects on skin protection.

Lutein and zeaxanthin have been widely studied and have shown diverse beneficial effects on human health, particularly on optimizing eye health (Ma, \& Lin 2010). The biological mechanisms for the protective effects of both carotenoids may include powerful blue-light filtering activities and antioxidant properties. A sufficient amount of both carotenoids can be present in the eye retina to act as an antioxidant in the tissue. Astaxanthin is also known to prevent peroxidation at the lipid membrane level with greater efficiency than all other known antioxidant compounds (Guerin et al., 2003). The potential health-promoting effect of astaxanthin is mainly dependent on its strong antioxidant activity. 
Lycopene is one of the most predominant carotenoids in human plasma and can show stronger antioxidant activity than $\beta$-carotene and $\alpha$-tocopherol (Di Mascio et al., 1989; Miller et al., 1996). In this respect, skin lycopene is more sensitive to UV light stress than $\beta$-carotene. However, the major mechanism of the antioxidant activity of lycopene remains unclear (Erdman et al., 2009) as there is an insufficient concentration of lycopene in the target tissues to demonstrate the in vivo antioxidant activity. Many chronic diseases, including cardiovascular disease, cancer, diabetes, eye diseases, and aging, are the result of long-term oxidative stress. Thus, there has been great interest in the antioxidant properties of carotenoids. Additionally, the physiological effects of carotenoids cannot be explained only by their antioxidant activity.

The in vivo antioxidant activity of carotenoids is not dependent on the chemical ability of carotenoids to quench ${ }^{1} \mathrm{O}_{2}$ and scavenge free radicals but on other factors, such as absorption level, bioavailability, and accumulation in the target organ. To demonstrate meaningful activity, considerable amounts of carotenoids or their metabolites should be present in the targets to protect cells, tissues and other structures such as lipoproteins against oxidative damage. Furthermore, not all of the biological activities ascribed to carotenoids must necessarily be linked to their ability to quench ${ }^{1} \mathrm{O}_{2}$ and scavenge free radicals. A variety of potential mechanisms of action that may be independent of their conventional antioxidant properties are likely important in preventing diseases (Chew, and Park, 2004; Erdman et al., 2009; Miyashita et al, 2011; Miyashita, and Hosokawa, 2017). Among these properties, the modulation of specific gene and protein expression in biological systems by carotenoids may explain their other biological effects.

\section{Conclusion}

Carotenoids are known to have a variety of biological effects such as provitamin A and antioxidant activities. Many studies have shown the positive correlation between carotenoid intake and a reduction of non-communicable disease risk. In these studies, the carotenoids mostly originated from vegetables and fruits. Alternatively, there have been relatively few studies on marine carotenoids. Marine carotenoids have unique chemical structures with funcional groups such as allene $(-\mathrm{C}=\mathrm{C}=\mathrm{C}-)$, acetylene $(-\mathrm{C} \equiv \mathrm{C}-)$, acetyl $\left(-\mathrm{O}-\mathrm{CO}-\mathrm{CH}_{3}\right)$, and hydroxylmethyl $\left(-\mathrm{CH}_{2} \mathrm{OH}\right)$, among others, which are rarely found in carotenoids from terrestrial plants. These structural characteristics of marine carotenoids may provide novel bioactives that act as strong antioxidants and reduce the risk of many types of non-communicable diseases.

To date, much attention has been paid to two marine carotenoids, astaxanthin and fucoxanthin. Astaxanthin is a major carotenoid present in marine animals and shows several beneficial health effects based on its strong antioxidant activity. Fucoxanthin, a major algal carotenoid, is known to show anti-obesity and antidiabetic effects. These effects are mainly based on mechanisms that are independent of its antioxidant activity. Although both carotenoids are now commercially applied, more studies will be needed to identify a wider range of their application, especially focusing on the effective stabilization method and a good source of both carotenoids. In addition, other marine carotenoids should be explored.

\section{References}

Abidov, M., Ramazanov, Z., Seifulla, R., and Grachev, S. (2010). The effects of Xanthigen ${ }^{\text {TM }}$ in the weight management of obese premenopausal women with non-alcoholic fatty liver disease and normal liver fat. Diabetes Obesity and Metabolism 12: 72-81.

Airanthi, M.K.W. A., Sasaki, N., Iwasaki, S., Baba, N., Abe, M., Hosokawa, M., and Miyashita, K. (2011). Effect of brown seaweed lipids on fatty acid composition and lipid hydroperoxide levels of mouse liver. Journal of Agricultural and Food Chemistry 59: 4156-4163.

Ambati, R.R., Phang, S.M., Ravi, S., and Aswathanarayana, R.G. (2014). Astaxanthin: sources, extraction, stability, biological activities and its commercial applications - a review. Marine Drugs 12: 128-152.

Azam, M.S., Choi, J., Lee, M.-S., and Kim, H.-R. (2017). Hypopigmenting effects of brown algae-derived phytochemicals: a review on molecular mechanisms. Marine Drugs 15: E297.

Beppu, F., Hosokawa, M., Yim, M.-J., Shinoda, T., and Miyashita, K. (2013). Down-regulation of hepatic stearoyl-CoA desaturase-1 expression by Fucoxanthin via leptin signaling in diabetic/obese KK- $A^{\mathrm{y}}$ mice. Lipids 48: 449-455.

Beutner, S., Bloedorn, B., Frixel, S., Blanco, I.H., Hoffman, T., Martin, H.D., Mayer, B., Noach, P., Ruck, C., Schimdt, M., Schülke, I., Sell, S., Ernst, H., Haremza, S., Seybold, G., Sies, H., Stahl, W., and Walsh, R. (2001). Quantitative assessment of antioxidant properties of natural colorants and phytochemicals; carotenoids, flavonoids, phenols and indigoids: the role of $\beta$-carotene in antioxidant functions. Journal of the Science of Food and Agriculture 81: 559-568.

Böhm, F., Edge, R., and Truscott, G. (2012). Interactions of dietary carotenoids with activated (singlet) oxygen and free radicals: potential effects for human health. Molecular Nutrition \& Food Research 56: 205-216.

Bossi, O., Gartsbein, M., Leitges, M., Kuroki, T., Grossman, S., and Tennenbaum, T. (2008). UV irradiation increases ROS production via PKCdelta signaling in primary murine fibroblasts. Journal of Cellular Biochemistry 105: 194-207.

Brestoff, J.R., and Artis, D. (2015). Immune regulation of metabolic homeostasis in health and disease. Cell 161: 146-160.

Camera, E., Mastrofrancesco, A., Fabbri, C., Picardo, M., Sies, H., and Stahl, W. (2008). Astaxanthin, canthaxanthin and $\beta$-carotene differently affect UVA-induced oxidative damage and expression of oxidative stress-responsive enzymes. Experimental Dermatology 18: 222-231.

Cantrell, A., McGarvey, D.J., Truscott, T.G., Rancan, F., and Bohm, F. (2003). Singlet oxygen quenching by dietary carotenoids in a model membrane Environment. Archives of Biochemistry and Biophysics 412: 47-54.

Chen, J.-T., and Kotani, K. (2016). Astaxanthin as a potential protector of liver function: a review. Journal of Clinical Medicine Research 8: 701-704.

Chew, B.P., and Park, J. (2004). Carotenoid action on the immune response. The Journal of Nutrition 134: 257S-261S.

Conn, P.F., Schalch, W., and Truscott, G.T. (1991). The singlet oxygen and carotenoid interaction. Journal of Photochemistry and Photobiology B: Biology 11: 41-47.

Cooper, D.A., Eldridge, A.L., and Peters, J.C. (1999). Dietary carotenoids and certain cancers, heart disease and age related macular degeneration: a review of recent research. Nutrition Reviews 57: 201-214.

Corinaldesi, C., Barone, G., Marcellini, F., Anno, A.D., and Danovaro, R. (2017). Marine microbial-derived molecules and their potential use in cosmeceutical and cosmetic products. Marine Drugs 15: E118.

Dembitsky, V.M., and Maoka, T. (2007). Allenic and cumulenic lipids. Progress in Lipid Research 46: 328-375.

Davies, B.H. (1985). Carotenoid metabolism in animals: a biochemist's view. Pure and Applied Chemistry 57: 679-684.

De Luca, L.M. (1991). Retinoids and their receptors in differentiation, embryogenesis, and neoplasia. FASEB Journal 5: 2924-2933.

Di Mascio, P., Kaiser, S., and Sies, H. (1989). Lycopene as the most efficient biological carotenoid singlet oxygen quencher. Archives of Biochemistry and Biophysics 274: 532-538.

Edge, R., McGarvey, D.J., and Truscott, T.G. (1997). The carotenoids as antioxidants- a review. Journal of Photochemistry and Photobiology B: Biology 41: 189-200.

Erdman, J.W., Ford, N.A., and Lindshield, B.L. (2009). Are the health attributes of lycopene related to its antioxidant function? Archives of Biochemistry and Biophysics 483: 229-235.

Fassett, R.G., and Coombes, J.S. (2009). Astaxanthin, oxidative stress, in- 
flammation and cardiovascular disease. Future Cardiology 5: 333342.

Focsan, A.L., Polyakov, N.E., and Kispert, L.D. (2017). Photo protection of haematococcus pluvialis algae by astaxanthin: unique properties of Astaxanthin deduced by EPR, optical and electrochemical studies. Antioxidants (Basel) 6: E80

Fredric, J.P., David, G.W., and Charles, L.C. (2008). Astaxanthin: a novel potential treatment for oxidative stress and inflammation in cardiovascular disease. American Journal of Cardiology 101: 58D-68D.

Fukuzawa, K., Inokami, Y., Tokumura, A., Terao, J., and Suzuki, A. (1998). Rate constants for quenching singlet oxygen and activities for inhibiting lipid peroxidation of carotenoids and $\alpha$-tocopherol in liposomes. Lipids 33: 751-756.

Galasso, C., Corinaldesi, C., and Sansone, C. (2017). Carotenoids from marine organisms: biological functions and industrial applications. Antioxidants (Basel) 6: E96.

Gammone, M.A., and D'Orazio, N. (2015). Anti-obesity activity of the marine carotenoid fucoxanthin. Marine Drugs 13: 2196-2214.

Gammone, M.A., Riccioni, G., and D'Orazio, N. (2015). Marine carotenoids against oxidative stress: effects on human health. Marine Drugs 13: 6226-6246.

Ganesan, P., Noda, K., Manabe, Y., Ohkubo, T., Tanaka, Y., Maoka, T., Sugawara, T., and Hirata, T. (2011). Siphonaxanthin, a marine algal carotenoids from green algae, effectively induces apoptosis in human leukemia (HL-60) cells. Biochimica et Biophysica Acta 1810: 497-503.

Goodwin, T.W. (1986). Metabolism, nutrition, and function of carotenoids. Annual Review of Nutrition 6: 273-297.

Gori, T., and Nzel, T.M. (2011). Oxidative stress and endothelial dysfunction: therapeutic implications. Annals of Medicine 43: 259-272.

Goto, S., Kogure, K., Abe, K., Kimata, Y., Kitahama, K., Yamashita, E., and Terada, H. (2001). Efficient radical trapping at the surface and inside the phospholipid membrane is responsible for highly potent antiperoxidative activity of the carotenoid astaxanthin. Biochimica et Biophysica Acta 1512: 251-258.

Grasa-López, A., Miliar-García, Á., Quevedo-Corona, L., Paniagua-Castro, N., Escalona-Cardoso, G., Reyes-Maldonado, E., and Jaramillo-Flores, M.-E. (2016). Undaria pinnatifida and fucoxanthin ameliorate lipogenesis and markers of both inflammation and cardiovascular dysfunction in an animal model of diet-induced obesity. Marine Drugs 14: E148.

Grimmig, B., Kim, S.-H., Nash, K., Bickford, P.C., and Shytle, R.D. (2017). Neuroprotective mechanisms of astaxanthin: a potential therapeutic role in preserving cognitive function in age and neurodegeneration. GeroScience 39: 19-32.

Guerin, M., Huntley, M.E., and Olaizola, M. (2003). Haematococcus astaxanthin: applications for human health and nutrition. Trends Biotechnology 21: 210-216.

Harms, M., and Seale, P. (2013). Brown and beige fat: development, function and therapeutic potential. Nature Medicine 19: 1252-1263.

Henriksen, E.J., Bourey, R.E., Rodnick, K.J., Koranyi, L., Permutt, M.A., and Holloszy, J.O. (1999). Glucose transporter protein content and glucose transport capacity in rat skeletal muscles. American Journal of Physiology 259: E593-E598.

Heo, S.-J., and Jeon, Y.-J. (2009). Protective effect of fucoxanthin isolated from Sargassum siliquastrum on UV-B induced cell damage. Journal of Photochemistry and Photobiology B: Biology 95: 101-107.

Higuera-Ciapara, I., Félix-Valenzuela, L., and Goycoolea, F.M. (2006). Astaxanthin: a review of its chemistry and applications. Critical Reviews in Food Science and Nutrition 46: 185-196.

Hirayama, O., Nakamura, K., Hamada, S., and Kobayasi, Y. (1994). Singlet oxygen quenching ability of naturally occurring carotenoids. Lipids 29: 149-150.

Hitoe, S., and Shimoda, H. (2017). Seaweed fucoxanthin supplementation improves obesity parameters in mildly obese Japanese subjects. Functional Foods in Health and Disease 7: 246-262.

Hosokawa, M., Miyashita, T., Nishikawa, S., Emi, S., Tsukui, T., Beppu, F., Okada, T., and Miyashita, K. (2010). Fucoxanthin regulates adipocytokine mRNA expression in white adipose tissue of diabetic/obese KK- $A^{\mathrm{y}}$ mice. Archives of Biochemistry and Biophysics 504: 17-25.

Hu, X., Li, Y., Li, C., Fu, Y., Cai, F., Chen, Q., and Li, D. (2012). Combination of fucoxanthin and conjugated linoleic acid attenuates body weight gain and improves lipid metabolism in high-fat diet-induced obese rats. Archives of Biochemistry and Biophysics 519: 59-65.

Hu, X., Tao, N., Wang, X., Xiao, J., and Wang, M. (2016). Marine-derived bioactive compounds with anti-obesity effect: A review. Journal of Functional Foods 21: 372-387.

Huang, J.J., Lin, S., Xu, W., and Cheung, P.C.K. (2017). Occurrence and biosynthesis of carotenoids in phytoplankton. Biotechnology Advances 35: 597-618.

Huang, S., and Czech, M.P. (2007). The GLUT4 glucose transporter. Cell Metabolism 5: 237-252.

Hussein, G., Nakagawa, T., Goto, H., Shimada, Y., Matsumoto, K., Sankawa, U., and Watanabe, H. (2007). Astaxanthin ameliorates features of metabolic syndrome in SHR/NDmcr-cp. Life Sciences 80: 522-529.

Ikeuchi, M., Koyama, T., Takahashi, J., and Yazawa, K. (2007). Effects of astaxanthin in obese mice fed a high-fat diet. Bioscience, Biotechnology, and Biochemistry 71: 893-899.

lizuka, M., Ayaori, M., Uto-Kondo, H., Yakushiji, E., Takiguchi, S., Nakaya, K., Hisada, T., Sasaki, M., Komatsu, T., Yogo, M., Kishimoto, Y., Kondo, K., and Ikewaki, K. (2012). Astaxanthin enhances ATP-binding cassette transporter A1/G1 expressions and cholesterol efflux from macrophages. Journal of Nutritional Science and Vitaminology (Tokyo) 58: 96-104.

Jackson, H., Braun, C.L., and Ernst, H. (2008). The chemistry of novel xanthophyll carotenoids. American Journal of Cardiology 101: 50D-57D.

Kaneko, M., Nagamine, T., Nakazato, K., and Mori, M. (2013). The anti-apoptotic effect of fucoxanthin on carbon tetrachloride-induced hepatotoxicity. The journal of Toxicological Sciences 38: 115-126.

Kang, S.I., Ko, H.C., Shin, H.S., Kim, H.M., Hong, Y.S., Lee, N.H., and Kim, S.J. (2011). Fucoxanthin exerts differing effects on 3T3-L1 cells according to differentiation stage and inhibits glucose uptake in mature adipocytes. Biochemical and Biophysical Research Communications 409: 769-774.

Kang, S.-I., Shin, H.-S., Kim, H.-M., Yoon, S.-A., Kang, S.-W., Kim, J.-H., Ko, H.C., and Kim, S.-J. (2012). Petalonia binghamiae extract and its constituent fucoxanthin ameliorate high-fat diet-induced obesity by activating AMP-activated protein kinase. J. Agric. Food Chem. 60: 3389-3395.

Kishimoto, Y., Yoshida, H., and Kondo, K. (2016). Potential anti-atherosclerotic properties of Astaxanthin. Marine Drugs 14: E35.

Konishi, I., Hosokawa, M., Sashima, T., Kobayashi, H., and Miyashita, K. (2006). Halocynthiaxanthin and fucoxanthinol isolated from Halocynthia roretzi induce apoptosis in human leukemia, breast and colon cancer cells. Comparative Biochemistry and Physiology, Part C 142 : 53-59.

Konishi, I., Hosokawa, M., Sashima, T., Maoka, T., and Miyashita, K. (2008). Suppressive effects of alloxanthin and diatoxanthin from Halocynthia roretzi on LPS-induced expression of pro-inflammatory genes in RAW264.7 cells. Journal of Oleo Science 57: 181-189.

Kotake-Nara, E., Kushiro, M., Zhang, H., Sugawara, T., Miyashita, K., and Nagao, A. (2001). Carotenoids affect proliferation of human prostate cancer cells. The Journal of Nutrition 131: 3303-3306.

Krinsky, N.I., and Yeum, K.J. (2003). Carotenoid-radical interactions. Biochemical and Biophysical Research Communications 305: 754-760.

Lawlor, S.M., and ÒBrien, N.M. (1995). Astaxanthin: antioxidant effects in chicken embryo fibroblasts. Nutrition Research 15: 1695-1704.

Lim, B.P., Nagao, A., Terao, J., Tanaka, K., Suzuki, T., and Takama, K. (1992). Antioxidant activity of xanthophylls on peroxyl radical-mediated phospholipid peroxidation. Biochimica et Biophysica Acta 1126: 178-184

Liu, Y., Liu, M., Zhang, X., Chen, Q., Chen, H., Sun, L., and Liu, G. (2016). Protective effect of fucoxanthin isolated from Laminaria japonica against visible light-induced retinal damage both in vitro and in vivo. Journal of Agricultural and Food Chemistry 64: 416-424.

Lyons, N.M., and O'Brien, N.M. (2002). Modulatory effects of an algal extract containing astaxanthin on UVA-irradiated cells in culture. Journal of Dermatological Science 30: 73-84.

Ma, L., and Lin, X.-M. (2010). Effects of lutein and zeaxanthin on aspects of eye health. Journal of the Science of Food and Agriculture 90: 2-12.

Maeda, H. (2015). Nutraceutical effects of fucoxanthin for obesity and diabetes therapy: a review. Journal of Oleo Science 64: 125-132.

Maeda, H., Hosokawa, M., Sashima, T., Funayama, K., and Miyashita, K. (2005). Fucoxanthin from edible seaweed, Undaria pinnatifida, 
shows antiobesity effect through UCP1 expression in white adipose tissues. Biochemical and Biophysical Research Communications 332: 392-397.

Maeda, H., Hosokawa, M., Sashima, T., Funayama, K., and Miyashita, K. (2007a). Effect of medium-chain triacylglycerols on anti-obesity effect of fucoxanthin. Journal of Oleo Science 56: 615-621.

Maeda, H., Hosokawa, M., Sashima, T., and Miyashita, K. (2007b). Dietary combination of fucoxanthin and fish oil attenuates the weight gain of white adipose tissue and decrease blood glucose in obese/ diabetic KK-AY mice. Journal of Agricultural and Food Chemistry 55: 7701-7706.

Maeda, H., Hosokawa, M., Sashima, T., Murakami-Funayama, K., and Miyashita, K. (2009). Anti-obesity and anti-diabetic effects of fucoxanthin on diet-induced obesity conditions in a murine model. Molecular Medicine Reports 2: 897-902.

Maeda, H., Hosokawa, M., Sashima, T., Takahashi, N., Kawada, T., and Miyashita, K. (2006). Fucoxanthin and its metabolite, fucoxanthinol, suppress sdipocyte differentiation in 3T3-L1 cells. International Journal of Molecular Medicine 18: 147-152.

Mascio, P.D., Murhy, M.E., and Sies, H. (1991). Antioxidant defense systems: the role of carotenoids, tocopherols, and thiols. American Journal of Clinical Nutrition 53: 194S-200S.

Maoka, T. (2011). Carotenoids in marine animals. Marine Drugs 9: 278293.

Matsuno, T. (2001). Aquatic animal carotenoids. Fisheries Sciences 67: 771-783.

Matsushita, Y., Suzuki, R., Nara, E., Yokoyama, A., and Miyashita, K. (2000). Antioxidant activity of polar carotenoids including astaxanthin- $\beta$ glucoside from marine bacterium on PC liposomes. Fisheries Sciences 66: 980-985.

McNulty, H.P., Byun, J., Lockwood, S.F., Jacob, R.F., and Mason, R.P. (2007). Differential effects of carotenoids on lipid peroxidation due to membrane interactions: $\mathrm{X}$-ray diffraction analysis. Biochimica et Biophysica Acta 1768: 167-174.

Miller, N.J., Sampson, J., Candeias, L.P., Bramley, P.M., and Rice-Evans, C.A. (1996). Antioxidant activities of carotenes and xanthophylls. FEBS Letters 384: 240-242.

Miyashita, K., Nishikawa, S., Beppu, F., Tsukui, T., Abe, M., and Hosokawa, M. (2011). Allenic carotenoid, fucoxanthin, as a novel marine nutraceutical from brown seaweed. Journal of Science and Food Agriculture 91: 1166-1174.

Miyashita, K., and Hosokawa, M. (2017). Fucoxanthin in the management of obesity and its related disorders. Journal of Functional Foods 36 195-202.

Mueller, E. (2016). Browning and graying: novel transcriptional regulators of brown and beige fat tissues and aging. Frontiers in Endocrinology (Lausanne) 7: 30.

Muradian, K.H., Vaiserman, A., Min, K.-J., and Fraifeld, V.E. (2015). Fucoxanthin and lipid metabolism: A mini review. Nutrition, Metabolism and Cardiovascular Diseases 25: 891-897.

Murakami, A., Nakashima, M., Koshiba, T., Maoka, T., Nishino, H., Yano, M., Sumida, T., Kim, O.K., Koshimizu, K., and Ohigashi, H. (2000). Modifying effects of carotenoids on superoxide and nitric oxide generation from stimulated leukocytes. Cancer Letters 149: 115-123.

Naguib, Y.M.A. (2000). Antioxidant activities of astaxanthin and related carotenoids. Journal of Agricultural and Food Chemistry 48: 11501154.

Namitha, K.K., and Negi, P.S. (2010). Chemistry and biotechnology of carotenoids. Critical Reviews in Food Science and Nutrition 50: 728-760.

Nedergaard, J., Golozoubova, V., Matthias, A., Asadi, A., Jacobsson, A., and Cannon, B. (2001). UCP1: the only protein able to mediate adaptive non-shivering thermogenesis and metabolic inefficiency. Biochimica et Biophysica Acta 1504: 82-106.

Nishikawa, S., Hosokawa, M., and Miyashita, K. (2012). Fucoxanthin promotes translocation and induction of glucose transporter 4 in skeletal muscles of diabetic/obese KK-A(y) mice. Phytomedicine 19 389-394.

Nishino, H. (1998). Cancer prevention by carotenoids. Mutation Research 402: 159-163.

Nomura, T., Kikuchi, M., Kubodera, A., and Kawakami, Y. (1997). Protondonative antioxidant activity of fucoxanthin with 1,1-diphenyl-2-pic- rylhydrazyl (DPPH). Biochem. Molecular Biology International 42: 361-370.

Okada, T., Nakai, M., Maeda, H., Hosokawa, M., Sashima, T., and Miyashita, K. (2008). Suppressive effect of neoxanthin on the differentiation of 3T3-L1 adipose cells. Journal of Oleo Science 57: 345-351.

Park, H.J., Lee, M.K., Park, Y.B., Shin, Y.C., and Choi, M.S. (2011). Beneficial effects of Undaria pinnatifida ethanol extract on diet-inducedinsulin resistance in C57BL/6J mice. Food and Chemical Toxicology 49: 727-733.

Polívka, T., and Frank, H.A. (2010). Molecular factors controlling photosynthetic light harvesting by carotenoids. Accounts of Chemical Research 43: 1125-1134.

Ranga Rao, A., Raghunath Reddy, R.L., Baskaran, V., Sarada, R., and Ravishankar, G.A. (2010). Characterization of microalgal carotenoids by mass spectrometry and their bioavailability and antioxidant properties elucidated in rat model. Journal of Agricultural and Food Chemistry 58: 8553-8559.

Riboli, E., and Norat, T. (2007). Epidemiiologic evidence of the protective effect of fruit and vegetables on cancer risk. American Journal of Clinical Nutrition 78: 559S-569S.

Riccioni, G. (2009). Carotenoids and cardiovascular disease. Current Atherosclerosis Reports 11: 434-439.

Rothwell, N.J., and Stock, M.J. (1979). A role for brown adipose tissue in diet-induced thermogenesis. Nature 281: 31-35.

Sachindra, N.M., Sato, E., Maeda, H., Hosokawa, M., Niwano, Y., Kohno, M., and Miyashita, K. (2007). Radical scavenging and singlet oxygen quenching activity of marine carotenoid fucoxanthin and its metabolites. Journal of Agricultural and Food Chemistry 55: 8516-8522.

Sachindra, N.M., Bhaskar, N., and Mahendrakar, N.S. (2005). Carotenoids in different body components of Indian shrimps. Journal of the Science of Food and Agriculture 85: 167-172.

Schmidt, R. (2004). Deactivation of $\mathrm{O}_{2}\left({ }^{1} \Delta_{\mathrm{g}}\right)$ singlet oxygen by carotenoids: internal conversion of excited encounter complexes. The Journal of Physical Chemistry A 118: 5509-5513.

Smith, R.E., and Horwitz, B.A. (1969). Brown fat and thermogenesis. Physiological Reviews 49: 330-425.

Stahl, W., and Sies, H. (2003). Antioxidant activity of carotenoids. Molecular Aspects of Medicine 24: 345-351.

Stonik, V., and Stonik, I. Low-molecular-weight metabolites from diatoms: structures, biological roles and biosynthesis. Marine Drugs 13: 3672 3709.

Suganuma, K., Nakajima, H., Ohtsuki, M., and Imokawa, G. (2010). Astaxanthin attenuates the UVA-induced up-regulation of matrixmetalloproteinase-1 and skin fibroblast elastase in human dermal fibroblasts. Journal of Dermatological Science 58: 136-142.

Sugawara, T., Ganesan, P., Li, Z., Manabe, Y., and Hirata, T. (2014). Siphonaxanthin, a green algal carotenoid, as a novel functional compound. Marine Drugs 12: 3660-3668.

Sugawara, T., Yamashita, K., Sakai, S., Asai, A., Nagao, A., Shiraishi, T., and Hirata, T. (2007). Induction of apoptosis in DLD-1 human colon cancer cells by peridinin isolated from the dinoflagellate, Heterocapsa triquetra. Bioscience, Biotechnology, and Biochemistry 71: 1069-1072.

Takashima, M., Shichiri, M., Hagihara, Y., Yoshida, Y., and Niki, E. (2012). Capacity of fucoxanthin for scavenging peroxyl radicals and inhibition of lipid peroxidation in model systems. Free Radical Research 46: $1406-1412$

Takeuchi, S. (2011). Carotenoids in algae: distributions, biosyntheses and functions. Marine Drugs 9: 1101-1108.

Terao, J. (1989). Antioxidant activity of $\beta$-carotene-related carotenoids in solution. Lipids 24: 659-661.

Yan, X., Chuda, Y., Suzuki, M., and Nagata, T. (1999). Fucoxanthin as the major antioxidant in Hijikia fusiformis, a common edible seaweed. Bioscience, Biotechnology, and Biochemistry 63: 605-607.

Yang, Y., Seo, J.M., Nguyen, A., Pham, T.X., Park, H.J., Park, Y., Kim, B., Bruno, R.S., and Lee, J. (2011). Astaxanthin-rich extract from the green alga haematococcus pluvialis lowers plasma lipid concentrations and enhances antioxidant defense in apolipoprotein E knockout mice. The Journal of Nutrition 141: 1611-1617.

Yim, M.-J., Hosokawa, M., Mizushina, Y., Yoshida, H., Saito, Y., and Miyashita, K. (2011). Suppressive effects of amarouciaxanthin A on 3T3-L1 adipocyte differentiation through down-regulation of PPARY and C/ 
EBP $\alpha$ mRNA expression. Journal of Agricultural and Food Chemistry 59: 1646-1652.

Yuan, J.-P., Peng, J., Yin, K., and Wang, J.-H. (2011). Potential health-promoting effects of astaxanthin: a high-value carotenoid mostly from microalgae. Molecular Nutrition \& Food Research 55: 150-165.

Varela, J.C., Pereira, H., Vila, M., and León, R. (2015). Production of carotenoids by microalgae: achievements and challenges. Photosynthesis Research 125: 423-436.

Visioli, F., and Artaria, C. (2017). Astaxanthin in cardiovascular health and disease: mechanisms of action, therapeutic merits, and knowledge gaps. Food \& Function 8: 39-63.

Willett, W.C. (2001). Diet and cancer: one view at the start of the millennium. Cancer Epidemiology, Biomarkers \& Prevention 10: 3-8.

Woo, M.-N., Jeon, S.-M., Kim, H.-J., Lee, M.-K., Shin, S.-K., Shin, Y.C., Park, Y.-B., and Choi, M.-S. (2010). Fucoxanthin supplementation improves plasma and hepatic lipid metabolism and blood glucose concentration in high-fat fed C57BL/6N Mice. Chemico-Biological Interactions 186: 316-322.

Woo, M.-N., Jeon, S.-M., Shin, Y.C., Lee, M.-K., Kang, M.A., and Choi, M.-
S. (2009). Anti-obese property of fucoxanthin is partly mediated by altering lipid-regulating enzymes and uncoupling proteins of visceral adipose tissue in mice. Molecular Nutrition \& Food Research 53: 1603-1611.

Wu, H., Niu, H., Shao, A., Wu, C., Dixon, B.J., Zhang, J., Yang, S., and Wang, Y. (2015). Astaxanthin as a potential neuroprotective agent for neurological diseases. Marine Drugs 13: 5750-5766.

Zhang, H., Tang, Y., Zhang, Y., Zhang, S., Qu, J., Wang, X., Kong, R., Han, C., and Liu, Z. (2015). Fucoxanthin: a promising medicinal and nutritional ingredient. Evidence-Based Complementary and Alternative Medicine 2015: 723515.

Zheng, J., Piao, M.J., Keum, Y.S., Kim, H.S., and Hyun, J.W. (2013). Fucoxanthin protects cultured human keratinocytes against oxidative stress by blocking free radicals and inhibiting apoptosis. Biomolecules \& Therapeutics 21: 270-276.

Zheng, J., Piao, M.J., Kim, K.C., Yao, C.W., Cha, J.W., and Hyun, J.W. (2014). Fucoxanthin enhances the level of reduced glutathione via the Nrf2mediated pathway in human keratinocytes. Marine Drugs 12: 42144230. 